\title{
Low color temperature artificial lighting can slow myopia development: long-term study using juvenile monkeys
}

\section{Yingzhou Hu}

Kunming Institute of Zoology, Chinese Academy of Sciences

\section{Hua Yang}

Institute of Semiconductors, Chinese Academy of Sciences

Hao Li (D lihao@mail.kiz.ac.cn)

Kunming Institute of Zoology, Chinese Academy of Sciences https://orcid.org/0000-0002-3160-8719

\section{Longbao Lv}

Kunming Primate Research Center, Chinese Academy of Sciences, Kunming, Yunnan

\section{Jing Wu}

Kunming Institute of Zoology, Chinese Academy of Sciences

\section{Zhu Zhu}

Kunming Institute of Zoology, Chinese Academy of Sciences

\section{Yuhua Zhang}

Kunming Institute of Zoology, Chinese Academy of Sciences

\section{Fang-Fang Yan}

Institute of Psychology, Chinese Academy of Sciences

\section{Shuhan Fan}

Institute of Psychology, Chinese Academy of Sciences

\section{Shuxiao Wang}

China Academy of Building Research

Jianping Zhao

China Academy of Building Research

\section{Qiang Qi}

Kunming Institute of Zoology, Chinese Academy of Sciences

\section{Changbing Huang}

Institute of Psychology, Chinese Academy of Sciences https://orcid.org/0000-0003-4694-0834

\section{Xintian Hu}

Kunming Institute of Zoology, Chinese Academy of Sciences

\section{Article}


Keywords: Myopia, correlated color temperature (CCT), ocular axis, LED, incandescent lamp, juvenile monkeys

Posted Date: October 15th, 2021

DOI: https://doi.org/10.21203/rs.3.rs-952597/v1

License: (c) (1) This work is licensed under a Creative Commons Attribution 4.0 International License. Read Full License

Version of Record: A version of this preprint was published at Zoological Research on January 1st, 2022. See the published version at https://doi.org/10.24272/j.issn.2095-8137.2021.401. 


\section{Abstract}

The incidence of myopia has been increasing rapidly in recent decades, suggesting that environmental factors, such as light, may be a leading cause. Correlated color temperature (CCT) is a commonly used index to quantify the spectral composition of light. Here, we used 32 juvenile monkeys and selected four kinds of light with typical but different CCTs to study the relationship between CCT and ocular axial elongation. After half a year of observation, elongation of the ocular axis under low-CCT light was lower than that under high-CCT light. As excessive axial elongation is the main cause of juvenile myopia, these results provide a new approach for the prevention of juvenile myopia and establish a scientific foundation for introducing appropriate lighting standards in schools.

\section{Introduction}

The incidence rate of myopia has been increasing rapidly in recent decades, suggesting that environmental factors, such as light, may be a leading cause ${ }^{1}$. With the evolvement of light-emitting diode (LED) technology and demand for energy efficiency, the spectral composition of artificial lighting has changed significantly, with an increase in short wavelength (e.g., blue) components and decrease in long wavelength (e.g., red) components ${ }^{2}$. Given the importance of the spectral composition of light to the occurrence and development of myopia ${ }^{3-5}$, its transformation in artificial lighting may be related to the increase in myopia incidence. To date, however, this hypothesis remains unexplored.

Correlated color temperature (CCT), which characterizes the proportions of different wavelength components in light, is commonly used to quantify the spectral composition of a light source. A high CCT usually indicates richness in short wavelength components, while a low CCT indicates dominance of long wavelength components. Regarding effects on human subjects, most studies have explored the impact of CCT on visual comfort and fatigue under different artificial lighting conditions ${ }^{6-12}$, with the relationship between CCT and myopia development yet to be clarified. No direct study on the relationship between daily lighting CCT and myopia development in animals either.

On the other hand, driven by energy-saving requirements, most venues and lit spaces are undergoing a low-to-high CCT transition, as reflected in the national lighting standards of many countries and the production practices of major lighting manufacturers ${ }^{13}$. This transition has even been integrated into myopia prevention systems, becoming the basis for schools and parents to choose study lights for students. This trend will likely continue in the coming years, despite the lack of solid scientific evidence and potential damage to vision ${ }^{3-5}$. Therefore, determining the impact of different CCTs on the occurrence and development of myopia under typical indoor illumination is critical.

Due to their close evolutionary proximity, rhesus monkeys share a similar visual system as human children and adolescents, with highly similar structures and functional organization. As such, we selected 32 juvenile rhesus macaques as study subjects to ensure valid extrapolation of the experimental results to humans. We also selected three kinds of LED lamps with typical CCTs $(3000,4000$, and $5000 \mathrm{~K})$ and a 
conventional incandescent lamp (IL, $2700 \mathrm{~K}$ ) as the experimental light sources (Fig. S1). We quantitatively studied the relationship between light CCT and ocular axial elongation, an important and reliable index for myopia development ${ }^{14}$. The 32 monkeys were divided into four groups of eight animals, one group for each lighting condition. All four lamps were panel lights of the same size, mounted on the ceiling, and hung about $1 \mathrm{~m}$ above the top of each cage (please see Methods for details). Careful adjustments were made so that the illumination (504 $\pm 9 \mathrm{~lx})$ at the center of each cage. Measurements of ocular axial length, corneal curvature radius, anterior chamber depth, and refractive status were performed on the monkeys before the experiment and then at regular intervals (every 28-32 days) after the experiment started. All data were collected between 12:00 pm and 16:30 pm on the testing day. After 6 months of continuous observations, our results clearly showed that, contrary to current understanding, ocular axial elongation under low CCT illumination was significantly slower than that under high CCT illumination.

\section{Results}

\section{Effects of LED light CCT on ocular axial elongation}

In this study, we demonstrated that LED lights with different CCTs had different effects on ocular axial growth in juvenile monkeys (Fig. $1 \mathrm{~A}, F_{2,45}=4.711, P=0.014$ ). The increase in ocular axial length under artificial light with low CCT (3000 K) $(0.26 \pm 0.08 \mathrm{~mm}$, mean \pm standard deviation (SD)) was significantly smaller than that under high ССТ $(0.35 \pm 0.11 \mathrm{~mm}$ under $4000 \mathrm{~K}, P=0.029 ; 0.35 \pm 0.09 \mathrm{~mm}$ under 5000 $\mathrm{K}, P=0.037$, post-hoc test with Bonferroni correction). Thus, lights with higher CCT induced greater elongation $(0.09 \mathrm{~mm})$ within half a year, with no differences found between the two high CCT conditions (Fig. 1A).

Our research also showed significant differences in the increasing trend of the ocular axial length during the illumination period among the three groups (Fig. 1B). Using generalized linear mixed models (GLMs), we found that CCT had a significant effect on the trend of ocular axial growth during the whole lighting period $\left(F_{2,141}=9.45, P<0.001\right)$. Paired comparative analysis of the GLMs further showed that the ocular axial growth rate in monkeys reared under lower CCT light (3000 K) was slower than that in monkeys reared under higher CCT light ( $P=0.0004$ for $4000 \mathrm{~K} ; P=0.0002$ for $5000 \mathrm{~K})$. In addition, there was no significant difference in the ocular axial growth rate under the two high CCT conditions $(P=0.776)$.

In conclusion, our research showed that compared with high-CCT lighting, low-CCT lighting slowed ocular axial growth in juvenile monkeys, and this effect was robust and stable over the entire observation period.

\section{Comparison with conventional incandescent lamp (IL) with similar CCT $(2700 \mathrm{~K})$ but different spectra}

As LEDs have a different spectrum from traditional lights, we speculated whether the above findings (i.e., smaller axial elongation under lower CCT) are specific to LEDs. Therefore, we studied the effects of a 
traditional IL, with a similar CCT $(2700 \mathrm{~K})$ but different spectrum as the $3000 \mathrm{~K}$ LED, on ocular axial development in juvenile monkeys (IL $2700 \mathrm{~K}$ group). After 6 months of IL exposure, ocular axial length increased by $0.27 \pm 0.07 \mathrm{~mm}$, very close to that following $3000 \mathrm{~K} \mathrm{LED}$ exposure (two-tailed independent $t$ tests: $t_{30}=0.135, P=0.893$; Fig. 2A). There was also no significant difference in the increasing trend of ocular axial length between the two conditions (GLMM: $F_{1,93}=0.286, P=0.594$; Fig. $2 \mathrm{~B}$ ). Thus, these results clearly demonstrate that artificial illumination with low-CCT light, either traditional ILs $(2700 \mathrm{~K})$ or modern LEDs (3000 K), can slow ocular axial growth, and this effect is largely independent of spectral composition (shape).

\section{Discussion}

In summary, compared with high-CCT light (4000 K, 5000 K), low-CCT light (2700 K, 3000 K) slowed ocular axial growth in juvenile monkeys, with similar results found for low-CCT light sources composed of different spectra. This effect was robust and stable over the entire observation period. As excessive ocular axial elongation is the main cause of juvenile myopia, this study presents a new approach for the prevention and treatment of juvenile myopia and provides a scientific foundation for establishing lighting standards in schools and classrooms and for reconsidering anti-myopia products in the lighting industry.

Currently, there is a lack of experimental data to establish appropriate lighting standards in schools, especially regarding specific CCT ranges. Our results are important to help understand the impact of CCT on the development of myopia in children and adolescents. In addition, this study provides guidance for setting lighting standards and taking appropriate actions to prevent or limit visual damage.

At present, most lamps on the market have a CCT higher than $4000 \mathrm{~K}$, which is also proposed as a suitable CCT in the lighting guidelines for educational buildings in the United States and Britain. However, based on our results, high-CCT lighting is not optimal. As such, we should not blindly pursue high CCT for lighting efficiency in places of learning (or elsewhere). Instead, attention should be paid to the use of lowCCT lighting to better protect eyesight.

\section{Declarations}

\section{Acknowledgments}

We would like to thank Mr. Zhengfei Hu for the daily care of the monkeys, and Mr. Shunlong Wang for his hard work on data collection. This work was supported in part by the Key-Area Research and Development Program of Guangdong Province (2019B030335001), Science and Technology Service Network Initiative (STS) Project of Chinese Academy of Sciences (E02E1801), National Key R \& D Program of China (2018YFA0801403), the Scientific Instrument Developing Project of the Chinese Academy of Sciences (CAS) (022006), the Strategic Priority Research Program of the Chinese Academy of Sciences (XDB32060200), the National Natural Science Foundation of China (81941014, 81771387, 31800901, 31960178), CAS "Light of West China" program, the Applied Basic Research Programs of 
Science and Technology Commission Foundation of Yunnan Province (202001AT070130), and National Research Facility for Phenotypic and Genetic Analysis of Model Animals, Kunming Institute of Zoology, Chinese Academy of Sciences.

\section{Author contributions}

Conceptualization: $\mathrm{XH}, \mathrm{CH}, \mathrm{YH}, \mathrm{HY}, \mathrm{QQ}, \mathrm{JZ}, \mathrm{SW}$

Methodology: HY, HY, XH, QQ, HL, JZ, SW

Investigation: YH, HY, HL, JW, ZZ, YZ, LL

Visualization: $\mathrm{YH}, \mathrm{HY}, \mathrm{HL}, \mathrm{XH}$

Funding acquisition: $\mathrm{XH}, \mathrm{QQ}, \mathrm{CH}, \mathrm{YH}, \mathrm{HY}, \mathrm{HL}$

Project administration: QQ, XH

Supervision: $\mathrm{XH}, \mathrm{LL}, \mathrm{CH}, \mathrm{JZ}$

Writing - original draft: $\mathrm{YH}, \mathrm{XH}, \mathrm{HY}$

Writing - review \& editing: $\mathrm{CH}, \mathrm{SW}, \mathrm{JZ}, \mathrm{XH}, \mathrm{HL}, \mathrm{YH}$

\section{Competing interests}

Authors declare that they have no competing interests.

\section{Methods}

1. Study subjects and animal welfare

Thirty-two healthy young rhesus macaques (Macaca mulatta) (16 male, 16 female) were purchased from the Kunming Primate Research Center, Chinese Academy of Sciences (Yunnan, China). The monkeys were 2.1-2.5 years old ( $2.35 \pm 0.03$; mean $\pm S D)$, thus approximating primary school-aged children (6-7.5 years) and mimicking the vulnerable stage of ocular development in children.

Before the experiment, all monkeys were reared in a semi-open indoor-outdoor environment under natural sunlight. Each colony had an outdoor stainless-steel cage (2.67 m length) connected to an indoor room ( $2.61 \mathrm{~m}$ wide, $2.46 \mathrm{~m}$ long, and $2.58 \mathrm{~m}$ high). The monkeys could enter and leave the outdoor cage and indoor room at will. All rearing and experimental procedures were reviewed and approved by the Institutional Animal Care and Use Committee (IACUC) of the Kunming Primate Research Center (Approval Number: IACUC20029) and were in strict compliance with the National Care and Use of Animals Guide approved by the National Animal Research Authority (China) and the National Institutes of Health Guide for the Care and Use of Laboratory Animals (USA). 


\subsection{Selection of experimental lights}

Correlated color temperature (CCT) describes the proportions of different wavelength components of a light source. Notably, the same CCT can originate from different spectra. To ensure data comparability among different lighting conditions, we selected a commercial LED light with a typical spectrum (Fig. S1A, LED $4000 \mathrm{~K}$ ) and generated two other LED lights with different CCTs by varying the ratio of their long and short wavelength components (Fig. S1A, LED $3000 \mathrm{~K}$ and LED $5000 \mathrm{~K}$ ). The resulting CCTs of these three groups mentioned above were $2883 \pm 30 \mathrm{~K}, 3803 \pm 14 \mathrm{~K}, 4740 \pm 13 \mathrm{~K}$, respectively. For light comparison, we selected a conventional incandescent lamp (IL) with a CCT of $2700 \mathrm{~K}$ (Fig. S1B). All four lights were panel lights of the same size $(60 \times 60 \mathrm{~cm})$ and were mounted on the ceiling about $1 \mathrm{~m}$ above each cage.

\subsection{Experimental implementation}

Before the start of the experiment, all monkeys underwent ophthalmic examination and collection of ocular baseline data (e.g., ocular axial length, keratometry radius, anterior chamber depth, refractive status). During examination, subjects with cataracts or other ophthalmic diseases were excluded. The remaining monkeys were then divided into four groups of eight animals according to their axial lengths, so that the mean values of axial length were comparable among groups (Table S1, $F_{3,60}=0.001, P$ $=1.00$ ). At the beginning of the experiment, the four groups were randomly assigned to four experimental rooms with pre-installed experimental lights. All animals were transferred to their rooms on the same day for single-cage housing (each cage was $0.8 \mathrm{~m}$ wide, $0.8 \mathrm{~m}$ long, and $0.8 \mathrm{~m}$ high). Each room housed eight single cages arranged in two columns along each side of the room. Careful adjustments were made so that the illumination (504 \pm 9 Ix; measured with SFIM-400, Everfine Corporation, Hangzhou, China) at the center of each cage. As the lamps were mounted right on top of each cage, light directly illuminated each cage from the top.

The light cycle was $12 \mathrm{~h}$ light/12 h dark per day, with lights on from 07:00 am-19:00 pm. Each animal was provided with free access to food and water, and they were inspected daily by experienced veterinarians.

We did not intervene with visual development of the monkeys but allowed it to develop naturally under artificial lighting in order to mimic the developmental process of the ocular axial in children under indoor activity conditions. During the experiment, illumination at the center of each cage was measured and adjusted at regular intervals (28-32 days) to maintain an illumination of $\sim 500 \mathrm{~lx}$.

\subsection{Data collection}

Measurements of ocular axial length, corneal curvature radius, anterior chamber depth, and refractive status were performed on the monkeys at regular intervals (every 28-32 days). All data were collected at 
the same time of day between $12: 00 \mathrm{pm}$ and $16: 30 \mathrm{pm}$.

For data collection, each monkey was first anesthetized with ketamine (intramuscular administration, 2025 mg/kg, Jiangsu Zhongmu Beikang Pharmaceutical Co., Ltd., China) and acepromazine maleate (intramuscular administration, 0.15-0.20 mg/kg, Shanghai Aladdin Biochemical Technology Co., Ltd., China). Ocular axial length, corneal curvature radius, and anterior depth were then measured using an optical biometer (IOL Master, Carl Zeiss Meditec, Germany). Immediately after these measurements, tropicamide phenylephrine eye drops were administered (Mydrin-p, Santen, Osaka, Japan) in each of the monkey's eyes 5 min apart for mydriatic and ciliary anesthesia, and refractive examination was performed 20 min later using a computerized autorefractor meter (KR-800, TOPCON Medical Systems Inc., Japan). Due to the similarity between monkey and human eyes, measurement of monkey eyes using optical biometry and computerized autorefractometry has been proven feasible and effective ${ }^{15-17}$.

\section{Statistical analysis}

After ocular measurements were complete, data from the two eyes of each monkey were pooled together after statistical analyses showed no significant differences between them. The ocular axial length, corneal curvature radius, and anterior chamber depth data before and after artificial illumination were analyzed and compared by one-way analysis of variance (ANOVA), followed by Bonferroni correction for post hoc tests. The change trends of ocular parameters over time were analyzed and compared using a generalized linear mixed model (GLMM). All statistical analyses were performed using SPSS Statistics v27.0 (IBM Corp., Armonk, NY, USA).

\section{Supplementary Information}

Table S1. Baseline data on age, ocular optical parameters, and refractive status of four experimental groups. There were no significant differences in baseline data among the four experimental groups or between the eyes of each monkey in each group.

\begin{tabular}{|c|c|c|c|c|c|c|c|c|}
\hline & \multicolumn{8}{|c|}{ Baseline } \\
\hline & \multicolumn{2}{|c|}{$\begin{array}{l}\text { Incandescent lamp } 2700 \mathrm{~K} \\
(844 \pm 38 \text { days, } n=8)\end{array}$} & \multicolumn{2}{|c|}{$\begin{array}{l}\text { LED } 3000 \mathrm{~K} \\
(865 \pm 13 \text { days, } n=8)\end{array}$} & \multicolumn{2}{|c|}{$\begin{array}{l}\text { LED } 4000 \mathrm{~K} \\
(860 \pm 38 \text { days, } \mathrm{n}=8)\end{array}$} & \multicolumn{2}{|c|}{$\begin{array}{l}\text { LED } 5000 \mathrm{~K} \\
(856 \pm 28 \text { days, } \mathrm{n}=8)\end{array}$} \\
\hline & OD & OS & OD & os & OD & OS & OD & OS \\
\hline \multicolumn{9}{|l|}{ Axial length } \\
\hline$($ mean $\pm S D, m m)$ & $18.83 \pm 0.55$ & $18.82 \pm 0.52$ & $18.83 \pm 0.56$ & $18.84 \pm 0.55$ & $18.82 \pm 0.96$ & $18.84 \pm 0.93$ & $18.82 \pm 0.40$ & $18.82 \pm 0.42$ \\
\hline \multicolumn{9}{|c|}{ Corneal curvature radius } \\
\hline$($ mean $\pm \mathrm{SD}, \mathrm{mm})$ & $6.51 \pm 0.23$ & $6.50 \pm 0.21$ & $6.49 \pm 0.26$ & $6.48 \pm 0.26$ & $6.46 \pm 0.27$ & $6.46 \pm 0.27$ & $6.47 \pm 0.11$ & $6.45 \pm 0.10$ \\
\hline \multicolumn{9}{|c|}{ Anterior chamber depth } \\
\hline$($ mean $\pm S D, m m)$ & $3.36 \pm 0.15$ & $3.38 \pm 0.17$ & $3.46 \pm 0.19$ & $3.44 \pm 0.15$ & $3.45 \pm 0.33$ & $3.44 \pm 0.35$ & $3.41 \pm 0.17$ & $3.42 \pm 0.23$ \\
\hline
\end{tabular}

Compared with the ocular axial length, changes in the anterior chamber depth in the LED $3000 \mathrm{~K}$, LED $4000 \mathrm{~K}$, and LED $5000 \mathrm{~K}$ groups were $0.002 \pm 0.110,0.062 \pm 0.109$, and $0.024 \pm 0.125 \mathrm{~mm}$, respectively (Fig. S2A). The ANOVA results showed that there were no differences in anterior chamber depth among the three LED lighting groups (ANOVA: $F_{2,45}=1.137, P=0.330$ ). During the whole illumination period, the three different LED lights also had no significant effect on the growth trend of anterior chamber depth 
(GLMM: $F_{2,140}=1.718, P=0.183$ ) (Fig. S2B). Compared with the increase in axial length, the increase in anterior chamber depth was minimal. Therefore, we believe that the increase in ocular axial length was mainly due to growth of the vitreous cavity.

The changes in the corneal curvature radius in the LED $3000 \mathrm{~K}$, LED $4000 \mathrm{~K}$, and LED $5000 \mathrm{~K}$ groups were $-0.004 \pm 0.036,0.019 \pm 0.069$, and $0.014 \pm 0.062 \mathrm{~mm}$, respectively. In addition, ANOVA showed that there was no difference in the changes in corneal curvature radius among the three LED groups (ANOVA: $F_{2,21}$ $=0.712, P=0.496)$ (Fig. S2C). During the whole illumination period, the three LED lights had no significant effect on the change trend in the corneal curvature radius (GLMM: $F_{2,140}=0.817, P=0.444$ ) (Fig. S2D). Similar results were obtained in the IL (2700 K) experiment (Fig. S3).

\section{References}

1. Morgan, I. G., Ohno-Matsui, K. \& Saw, S.-M. Myopia. The Lancet 379, 1739-1748 (2012).

2. Behar-Cohen, F. et al. Light-emitting diodes (LED) for domestic lighting: Any risks for the eye? Progress in retinal and eye research 30, 239-257 (2011).

3. Guggenheim, J. A. et al. Time outdoors and physical activity as predictors of incident myopia in childhood: a prospective cohort study. Investigative ophthalmology \& visual science $53,2856-2865$ (2012).

4. He, M. et al. Effect of time spent outdoors at school on the development of myopia among children in China: a randomized clinical trial. Jama 314, 1142-1148 (2015).

5. Lingham, G., Mackey, D. A., Lucas, R. \& Yazar, S. How does spending time outdoors protect against myopia? A review. British Journal of Ophthalmology 104, 593-599 (2020).

6. Davis, R. G. \& Ginthner, D. N. Correlated color temperature, illuminance level, and the Kruithof curve. Journal of the Illuminating Engineering Society 19, 27-38 (1990).

7. Knez, I. Effects of colour of light on nonvisual psychological processes. Journal of environmental psychology 21, 201-208 (2001).

8. Lucas, R. J. et al. Measuring and using light in the melanopsin age. Trends in neurosciences 37, 1-9 (2014).

9. Keis, O., Helbig, H., Streb, J. \& Hille, K. Influence of blue-enriched classroom lighting on students $\square$ cognitive performance. Trends in Neuroscience and Education 3, 86-92 (2014).

10. Zhu, Y. et al. Effects of illuminance and correlated color temperature on daytime cognitive performance, subjective mood, and alertness in healthy adults. Environment and Behavior 51, 199230 (2019).

11. Lin, J. et al. Several biological benefits of the low color temperature light-emitting diodes based normal indoor lighting source. Scientific reports 9, 1-8 (2019).

12. Lan, L., Hadji, S., Xia, L. \& Lian, Z. The effects of light illuminance and correlated color temperature on mood and creativity. Building Simulation 14, 463-475, doi:10.1007/s12273-020-0652-z (2021). 
13. Cai, J. et al. Influence of LED correlated color temperature on ocular physiological function and subjective perception of discomfort. leee Access 6, 25209-25213 (2017).

14. Rucker, F. Monochromatic and white light and the regulation of eye growth. Experimental eye research 184, 172-182 (2019).

15. Hung, L.-F., Ramamirtham, R., Wensveen, J. M., Harwerth, R. S. \& Smith III, E. L. Objective and subjective refractive error measurements in monkeys. Optometry and vision science: official publication of the American Academy of Optometry 89, 168 (2012).

16. Ostrin, L. A., Hung, L.-F., Smith III, E. L. \& Wildsoet, C. F. Lamina Cribrosa Geometry and Axial Length in Normal Rhesus Monkeys. Investigative Ophthalmology \& Visual Science 53, 3469-3469 (2012).

17. Lin, R. et al. Screening of high myopia in non-human primates. Investigative Ophthalmology \& Visual Science 60, 5870-5870 (2019).

\section{Figures}
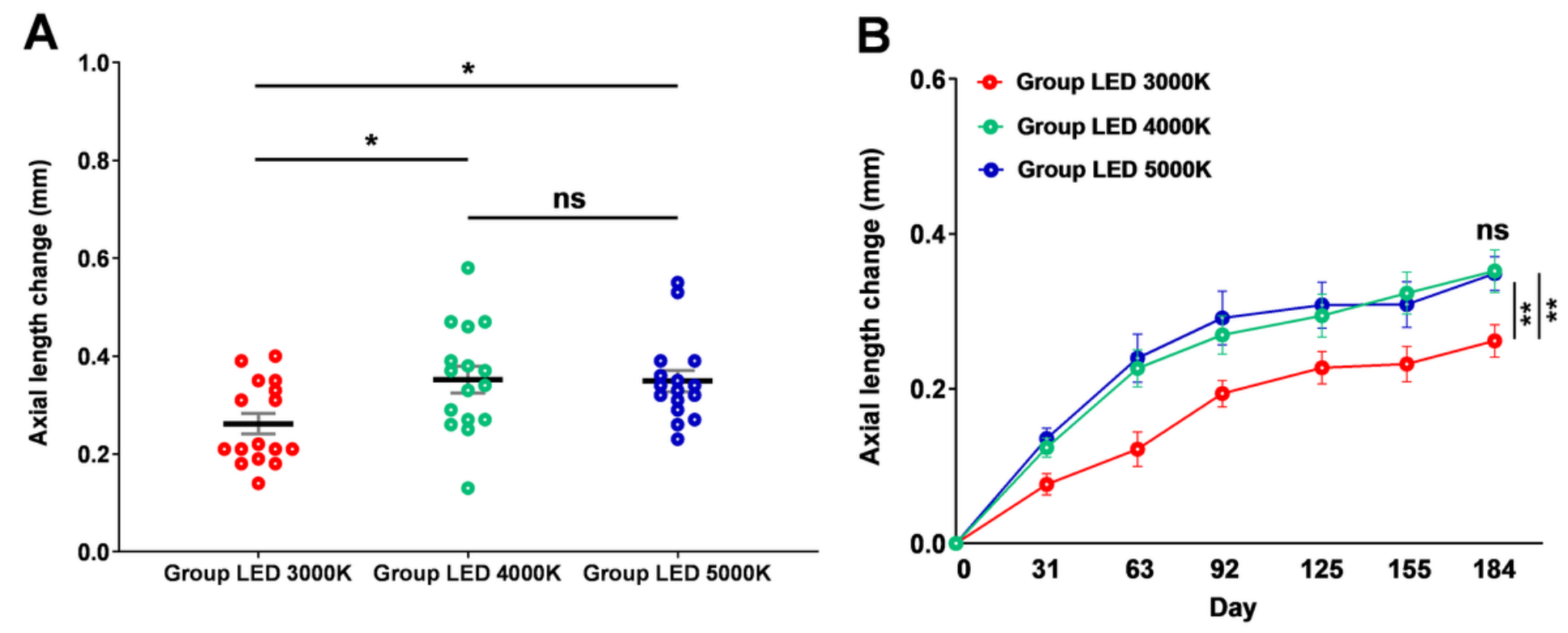

\section{Figure 1}

Increased ocular axial length in three juvenile monkey groups under different CCT LED lighting for 184 days. (A) LED $3000 \mathrm{~K}$ group (red circles) showed the lowest increase in ocular axial length, compared with the LED $4000 \mathrm{~K}$ (green circles) and LED $5000 \mathrm{~K}$ groups (blue circles). There was no significant difference between LED $4000 \mathrm{~K}$ and LED $5000 \mathrm{~K}$ monkeys. (B) Increased ocular axial length over time in three different LED lighting conditions. Ocular axial growth rate under artificial light with lower CCT (e.g., 3000 $\mathrm{K}$; red circles) was significantly smaller than that under light with higher CCTs (e.g., $4000 \mathrm{~K}$, green circles; $5000 \mathrm{~K}$, blue circles). Differences in growth rates between the two higher CCT conditions were not significant (ns). Each dataset contained 16 data points (two eyes in each monkey). *: $\mathrm{P}<0.05$; ** $\mathrm{P}<$ 0.01 . Data are mean \pm standard error of the mean (SEM). 

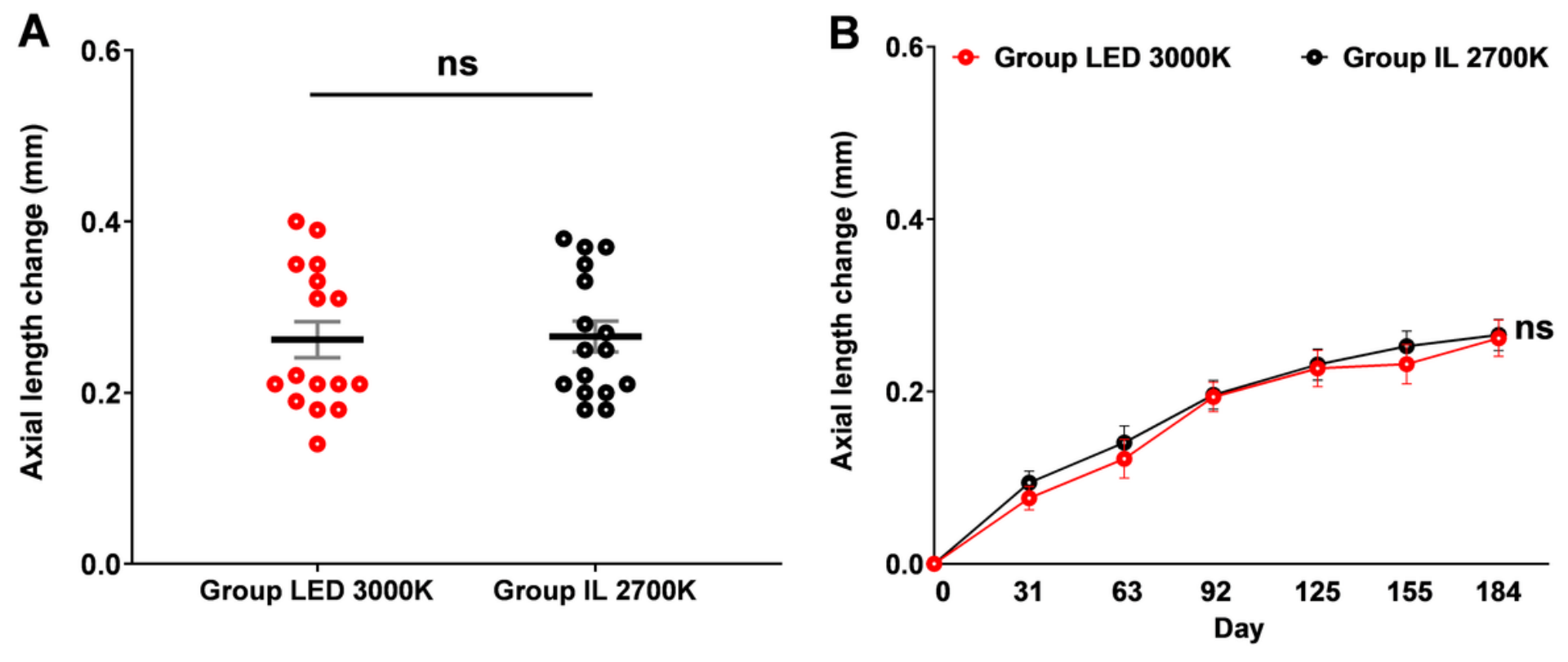

Figure 2

Effects of conventional incandescent lamp (2700 K; black circles) and LED with comparable CCT (3000 $\mathrm{K}$; red circles) on development of ocular axial length in juvenile rhesus macaques. Statistical analysis found no significant differences in ocular axial growth between the two groups $(A)$ or in axial growth trends throughout the experiment (B). Data are mean \pm SEM.
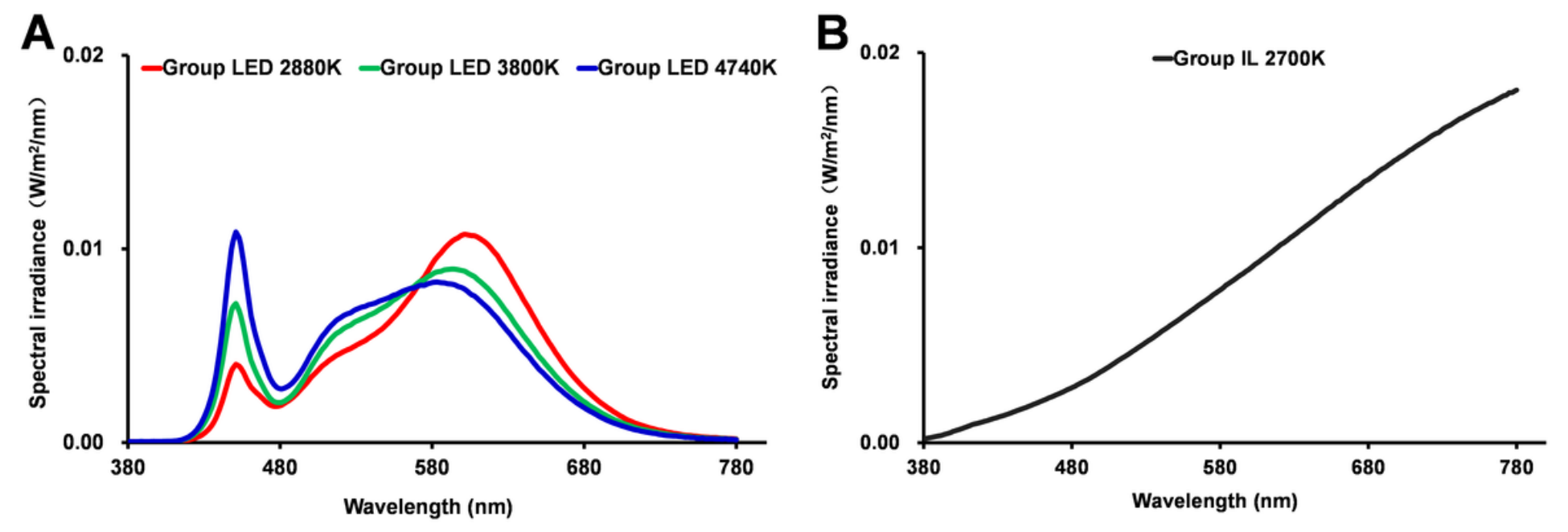

Figure 3

Fig. S1. Spectral irradiance curves of four lights used in this study (measured by a spectral flicking irradiance meter SFIM-400, Everfine Corporation, Hangzhou, China). (A) Spectral irradiance curves of three common commercial LEDs used in this study, which showed similar spectral profiles. Due to differences in the ratio of blue and yellow green light bands in each light, CCT increased with increasing blue light band ratio. CCTs of the three groups were $2883 \pm 30,3803 \pm 14$, and $4740 \pm 13 \mathrm{~K}$, respectively. (B) The spectral irradiance curve of an incandescent lamp (CCT: $2709 \pm 74 \mathrm{~K}$ ). 

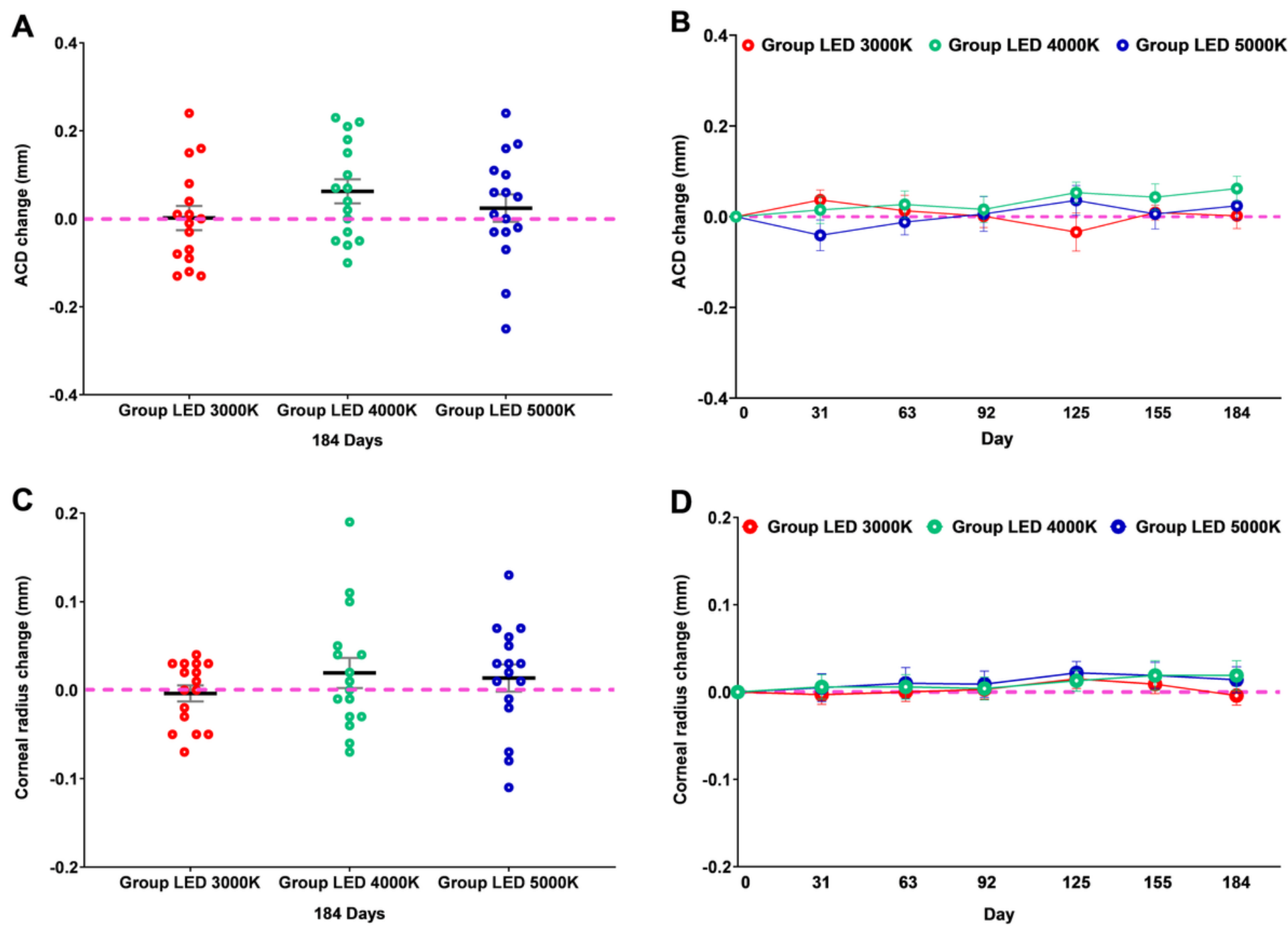

Figure 4

Fig. S2. Changes and trends in anterior chamber depth (ACD) as well as corneal curvature radius in three groups of juvenile monkeys under different LED lighting exposure. There were no significant differences in ACD and corneal curvature radius among the three groups. $(A)$ and $(C)$ Changes in ACD and corneal curvature radius, respectively, at time of last measurement. (B) and (D) Trends in ACD and corneal curvature radius, respectively, throughout light period. Each dataset contains 16 data points (two eyes from each monkey). Data are mean \pm SEM. 

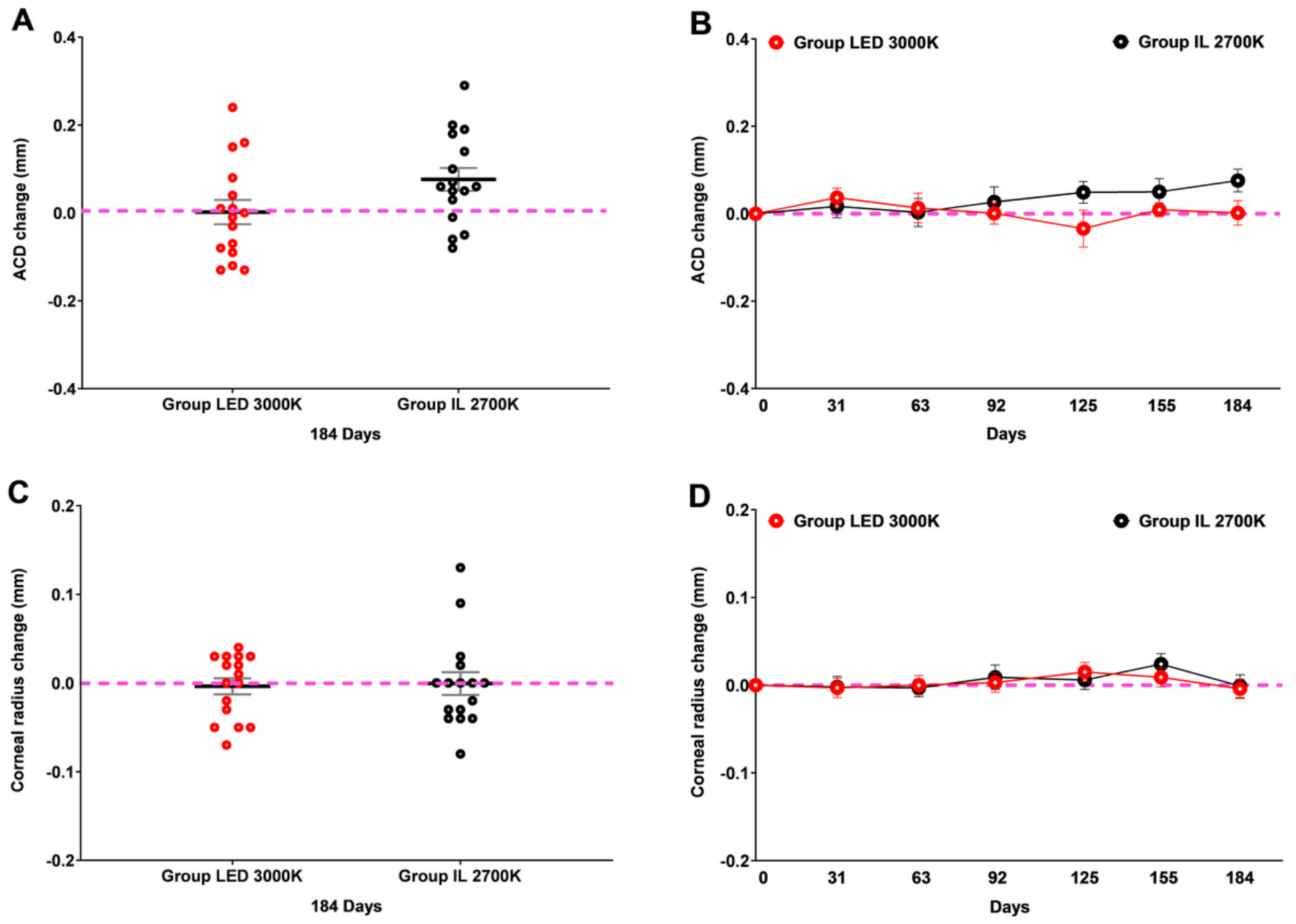

Figure 5

Fig. S3. Effects of conventional incandescent lamp (IL, $2700 \mathrm{~K}$ ) and LED $3000 \mathrm{~K}$ light exposure on anterior chamber depth (ACD) (A, B) and corneal curvature radius in juvenile monkeys $(C, D)$. There were no significant differences in $A C D$ and corneal curvature radius between groups. $(A)$ and $(C)$ Changes in ACD and corneal curvature radius, respectively, at time of last measurement. (B) and (D) Trends in ACD and corneal curvature radius, respectively, throughout light period. Data are mean \pm SEM. 